\title{
Intrapartum ultrasound use in clinical practice as a predictor of delivery mode during prolonged second stage of labor
}

tamar katzir ( $\square$ tamar.katzir1@mail.huji.ac.il )

Kaplan Medical Center https://orcid.org/0000-0002-2626-9070

\section{Yoav Brezinov}

Hebrew University of Medicine https://orcid.org/0000-0001-6452-9868

Ella Kharish

Hebrew University of Medicine https://orcid.org/0000-0001-6350-4546

\section{Shira Hadad}

Hebrew University of Medicine

\section{Edi Vaisbuch}

Hebrew University of Medicine https://orcid.org/0000-0002-8400-9031

Roni Levy ( $\nabla$ roni_l@clalit.org.il )

Hebrew University of Medicine https://orcid.org/0000-0003-2618-6328

\section{Research Article}

Keywords: Intrapartum ultrasound (IPUS), Angle of progression (AOP), pregnancy, nulliparous, prolonged second stage, mode of delivery

Posted Date: July 14th, 2021

DOl: https://doi.org/10.21203/rs.3.rs-596945/v1

License: (9) This work is licensed under a Creative Commons Attribution 4.0 International License.

Read Full License 


\section{Abstract \\ Purpose}

To determine the validity of Intrapartum ultrasound (IPUS), and particularly the angle of progression $(\mathrm{AOP})$, in predicting delivery mode when measured in real-life clinical practice among women with protracted second stages of labor.

\section{Methods}

Using electronic medical records, nulliparous women with a second stage of labor of $\geq 3$ hours ("prolonged") and a documented AOP measurement during the second stage were identified. The ability of a single AOP measurement in "prolonged" second stage to predict a vaginal delivery (VD) was assessed. Fetal head descend, measured by AOP change/hour (calculated from serial measurements) was compared between women who delivered vaginally and those who had a cesarean delivery (CD) for arrest of descent.

\section{Results}

Of the 191 women who met the inclusion criteria, 62 (32.5\%) delivered spontaneously, 96 (50.2\%) had a vacuum extraction (VE) and $33(17.3 \%)$ had a CD. The mean AOP was wider among women who had VD (spontaneous or VE) compared to those who had CD $\left(153^{\circ} \pm 19\right.$ vs. $\left.133^{\circ} \pm 17, p<0.001\right)$. Wider AOPs were associated with higher rates of VD and an AOP $\geq 127^{\circ}$ was associated with a VD rate of $88.6 \%$ (148/167). Among the 87 women who had more than one AOP measurement, the mean AOP change per hour was higher in the VD group than in the CD group $\left(15.1^{\circ} \pm 11.4^{\circ}\right.$ vs. $\left.6.2^{\circ} \pm 6.3^{\circ}, p<0.001\right)$.

\section{Conclusion}

Ultrasound assessed fetal head station in nulliparous women with a protracted second stage of labor can be an accurate and objective additive tool in predicting mode and interval time to delivery in real-life clinical practice.

\section{Introduction}

The second stage of labor's maximal duration time is a controversial issue. While prolonging the second stage may increase the chance of vaginal delivery (VD), there is concern about potential increase rates of adverse maternal and/or neonatal outcomes [1]. In 2014 the American College of Obstetricians and Gynecologists (ACOG) and the Society of Maternal and Fetal Medicine (SMFM) endorsed changing the definition of prolonged second stage of labor, stating that there is no specific maximal length of the second stage of labor as long as progress is being observed [2]. Yet, after adopting this change in 
definition some reported an increased maternal and neonatal morbidity [3, 4]. Additionally, cesarean deliveries (CD), when performed in advanced second stages of labor, are associated with a higher complications rate [5-7]. Therefore, accurate predictions of the likelihood of VD for women with a prolonged second stage is essential to minimize potential complications. The classical way to assess labor progress and fetal head station and position and to predict delivery mode is by digital vaginal examination. However, this method is subjective and inaccurate $[8,9]$ and thus not optimal for the significant decisions required during the most important phase of pregnancy, the delivery.

During the last decade, intrapartum ultrasound (IPUS) played an essential role in the management of labor [10-23]. IPUS was found to be more accurate in determining fetal head position [24-28], station [29-31] and descent [32-39], as well as in estimating the interval time to delivery [40-42]. In 2018, the International Society of Ultrasound in Obstetrics and Gynecology (ISUOG) issued guidelines recommending to performed IPUS in any delay in the second stage of labor [43].

Several studies evaluated the role of IPUS in predicting the chance of VD in women with a prolonged second stage of labor [30,44-47]. Following these reports, IPUS for assessment of fetal head position and station (by the AOP) in women with a protracted second stage has been adopted and commonly performed in our labor ward to assist in clinical decisions. Yet, the utility of IPUS for these purposes, outside of research settings, has not been well studied. Thus, the aim of this study was to determine, whether, IPUS and particularly the AOP when measured and used in real-life clinical practice by "average" labor ward physicians, can be a useful tool in predicting mode and interval time to delivery among women with a slow progress in the second stage of labor.

\section{Study Design}

This is a retrospective cohort study including women who delivered at Kaplan Medical Center from January 2017 to April 2020. We searched our electronic medical records for nulliparous women with a viable singleton pregnancy at term (>37 completed weeks), who received neuraxial analgesia and had a second stage of labor of at least 3 hours (defined as "prolonged" second stage of labor for the purpose of this study), and for whom an AOP measurement was documented at 2:30 to 3:30 hours from complete dilatation diagnosis. This timing was chosen since in real life situations, the required assessment (per our protocol) at 3 hours from complete dilatation is hard to implement at the exact timing. Women who had a vacuum extraction (VE) or a CD during the second stage of labor solely for a non-reassuring fetal heart rate were excluded from the study.

In our labor ward, IPUS is commonly used by the clinical staff as an additive tool to the digital exam. Before using this modality, all physicians designated to take care of laboring women, both during daytime and nighttime shifts, were required to complete training sessions and to be qualified by the director of the Labor \& Delivery ward (RL) before independently performing IPUS. The use of US during the second stage of labor was performed at the discretion of the clinical staff on duty. Currently, for the decision-making process, we commonly assess fetal head position, station and descent at about three hours from 
complete dilatation, both by digital vaginal examination and US and the decision whether to perform operative delivery or allow more time is based on the clinical judgement after integrating the available information.

Demographic and clinical data were retrieved from the electronic medical records. The Institutional Review Board of the Kaplan Medical Center approved the study protocol (KMC0206-19).

\section{Intrapartum Ultrasound assessment}

Our labor ward is equipped with portable US machines with a 2-5-MHz transabdominal two-dimensional convex transducer (Voluson P6, General Electric, Zift, Austria). Fetal head position is acquired by transabdominal US according to ISUOG guidelines [43]. For the AOP measurements, the transducer is covered with a glove and placed transperineally between the labia in a midsagittal view and the angle is measured between the long axis of the pubic bone and a line drawn from the lowest edge of the pubis to the deepest bony part of the fetal skull se described by Barbera et al. [10]. US parameters are documented in the patient electronic medical chart.

\section{Statistical analysis}

The mean AOP at prolonged second stage was compared between women who had a VD (spontaneous or $\mathrm{VE}$ ) to those who had a $\mathrm{CD}$. For delivery mode comparisons we used the head station conversion table from fetal head station (in cm) to AOP as described by Tutchek et al. [40] (AOP 127 $, 138^{\circ}, 148^{\circ}$, correspond to head station 1, 2, 3 respectively). Among women with more than one AOP measurement during the second stage of labor, the pace of labor progress was compared between women with prolonged second stage of labor who had VD and those who eventually had a CD.

Demographic and clinical characteristics were summarized using mean and standard deviation or median (minimum, maximum) for continuous variables and frequency tables for categorical variables. Continuous variables were tested for normality using Shapiro wilk test and compared between the study groups using T-test for normally distributed variables. Categorial variables were compared using Pearson Chi-square test. A logistic regression model containing demographic data (age, BMI, gestational age and birthweight), clinical data (labor induction, oxytocin, and second stage duration) and AOP measurements was generated. The variables were tested for statistical significance prediction of VD. Kaplan-Meier curves were used to assess the association between AOP groups $\left(<127^{\circ}, 127^{\circ}-137^{\circ}\right.$ and $\left.138^{\circ}-147^{\circ}\right)$ and the interval time (minutes) to delivery. The groups were compared using a log-rank test, and cases were censored at the time of CD. A p value $<0.05$ was considered statistically significant. Data were analyzed using the statistical software package IBM SPSS Statistics version 25.0.

\section{Results}

During the study period 5,266 (27.3\%) nulliparous women delivered in our medical center. Among them, $312(5.9 \%)$ had a prolonged second stage, (Fig. 1). Of the 191 (61\%) women who met all the inclusion 
criteria; 158 (82.7\%) delivered vaginally [62 (32.5\%) spontaneously and 96 (50.2\%) had a VE] and 33 (17.3\%) underwent a CD. One of 97 VE attempts had failed (1\%). Of note, in our center, forceps deliveries are not performed and the rates of VE and CD among nulliparous women are about $13 \%$ and $15 \%$, respectively. The demographic and clinical characteristics of the study population are described in Table 1. The BMI, the rates of oxytocin augmentation and induction of labor, as well as US assessed OP position were significantly higher among women who had a CD compared to those who delivered vaginally.

The mean AOP at 2:30 to 3:30 hours from complete dilatation was wider among women who had a VD than that of those who eventually had a $C D\left(153^{\circ} \pm 19\right.$ vs. $133^{\circ} \pm 17$, respectively; $p<0.001$, Fig. 2$)$.

Figure 3 depicts the association between the rate of VD and the different AOP cutoffs as suggested by the conversion table from fetal head station described by Tutchek et al. [40]. A wider AOP was associated with higher rates of VD and an AOP $\geq 127^{\circ}$ was associated with a VD rate of $88.6 \%(148 / 167)$.

An OP-position as assessed by US $(30 / 191,15.7 \%)$ was associated with a narrower mean AOP at 2:30 3:30 hours from completed dilatation than a non-OP position $\left(142.2^{\circ} \pm 17^{\circ}\right.$ vs. $151.1^{\circ} \pm 20^{\circ}$, respectively; $p$ $<0.013)$. The VD rate among these women was only $50 \%(n=15)$ and the mean AOP was wider in women who had VD compared to those who had CD $\left(150.2^{\circ} \pm 3^{\circ}\right.$ vs. $\left.134.3^{\circ} \pm 2^{\circ}, \mathrm{p}<0.0017\right)$.

Since an US assessed OP-position at prolonged second stage was associated with a high CD rate regardless of the AOP, we included in the multivariable logistic regression analysis only women with fetuses in a non-OP position (Table 2). The model revealed that, in addition to the neonatal birthweight and labor induction rate, the AOP was independently associated with the mode of delivery (OR 1.07, 95\% Cl 1.03-1.11, $p<0.001)$.

Additional AOP measurements (one to four) during the second stage of labor were available for 87 women. Among these women, the mean AOP change per hour was significantly higher in women who delivered vaginally $(n=66)$ compared to those $(n=21)$ who had a CD $\left(15.1^{\circ} \pm 11.4\right.$ vs. $5.7^{\circ} \pm 6.1^{\circ}$, respectively; $p<0.001$, Fig. 4). The individual slope patterns of the change in AOP during the second stage of labor for women who had a VD and for those who underwent CD are illustrated in Fig. 5.

A Kaplan-Meier plot for the time interval from AOP measurements at 2:30 - 3:30 hours from complete dilatation to VD was analyzed according to three AOP cut-offs: $<127^{\circ}, 127^{\circ}-137^{\circ}$ and $138^{\circ}-147^{\circ}(\log$ rank test, $p<0.001$ ). Among these groups, the wider was the AOP, the shorter was the remaining time (to delivery ( $95 \pm 8.8,72 \pm 5.6,56 \pm 6.6$ minutes, respectively), Fig. 7 .

\section{Discussion}

The principal finding of this study is that the AOP, as measured in routine clinical practice settings by the "average" labor ward physician, can assist in predicting the likelihood of a VD and in estimating the remaining time to birth in nulliparous women with neuraxial analgesia and a prolonged second stage of 
labor. Our study reaffirm the results of previous prospective studies, performed by physicians with expertise in US imaging and a particular interest in IPUS [10,30,34,36,44,46], addressing the role of IPUS and particularly the AOP in managing protracted second stage of labor.

While several studies investigated the role of IPUS for predicting successful instrumental deliveries, only a few studied the role of US in decision making in nulliparous women with slow fetal head descend during the second stage of labor [15, 30,44-47]. Kalache et al. [44] in a pioneering study have reported that a cutoff AOP of $120^{\circ}$ predicted a VD in $90 \%$ of 26 nulliparous women with a prolonged second stage and a fetus in the OA position. On the other hand, Chan et al., [30] on a population of Chinese women without epidural and a prolonged second stage, found that a cutoff of $138^{\circ}$ accurately predicted a successful VD. Dall'Asta et al. [46] found that among other parameters, the AOP at the acme of the pushing effort was wider in women who pushed for more than two hours and eventually had a spontaneous VD compared to those who ended with an operative delivery. Although, additional US parameters have been suggested to predict the mode of delivery in women with a delay in the second stage of labor, such as head direction [47], head symphysis angle [46], and midline angle [46], we could not address their utility in this study as these methods are difficult to be to applied by the "average" labor ward staff as they require more experience and advanced US skills. Indeed the ISUOG recommended the AOP as one of the primary sonographic parameter suitable for assessing fetal head progress, especially during prolonged second stage of labor, and our study confirms the feasibility of endorsement of this recommendation [43].

The consensus of the ACOG from 2014 states that there is no exact limit to the length of the second stage of labor as long as there is a progress in fetal head descend, and highlights the paramount importance of accurately assessing fetal head descend in the birth canal [2]. When the second stage is prolonged, the birth attendant needs to balance the risks and benefits to the mother and her neonate by allowing more time toward a VD on the one hand, to those associated with an immediate instrumental or $\mathrm{CD}$ on the other. In accordance with a recent study prospectively performed in a research setting by two SMFM experts [48], we have shown the feasibility of IPUS and particularly the AOP to be used in routine settings for diagnosing abnormal fetal head descend and to differentiate between women with a high chance to deliver vaginally and those at high risk of having a CD.

Regarding the interval time to delivery, in accordance to other prospective studies [36, 41, 42], we demonstrated that in nulliparous women there is a significant correlation between the US-assessed fetal head station at prolonged second stage and the remaining time to delivery. However, our study's retrospective nature precludes us from providing a reliable estimation of the remaining time to delivery according to the AOP measurement since there is no uniformity in the clinical management of the second stage among physicians.

The strengths of this study are the use of IPUS in a relatively large cohort of a homogeneous population of nulliparous women with neuraxial analgesia and a prolonged second stage of labor, where most of the significant clinical decisions during labor are taken. Furthermore, to the best of our knowledge, this is the first study to test the performance of IPUS in "real-life" clinical practice, a setting where most clinical staff 
on duty lack extended US skills, as in most delivery rooms worldwide. Our study has several limitations. First, this was a retrospective descriptive study thus the clinicians were obviously not blinded to the US measurements. Second, not all nulliparous women with a prolonged second stage of labor were included in the study as a documented AOP was available for $63 \%$ of the cohort thus a there is a potential for a selection bias.

To conclude, our findings demonstrate that IPUS can be safely implemented in routine real-life clinical practice as an additional tool to guide physicians in the important clinical making decisions during a protracted second stage of labor. The AOP in an objective and accurate US parameter for assessing fetal head station and progress in the birth canal, even when performed in clinical rather in research settings.

\section{Declarations}

\section{Authors' contributions}

T. Katzir: Data collection, Data analysis, Manuscript writing. E. Vaisbuch: Project development, Protocol development, Data analysis, Manuscript editing. Y. Brezinov: Data collection. E. Harish: Data collection. S. Hadad: Data collection. R. Levy: Project development, Protocol development, Data analysis, Manuscript editing

\section{Declaration}

The authors report no conflicts of interest in this work.

\section{Ethics approval}

Ethical approval was waived by the local Ethics Committee of Kaplan Medical Center. In view of the retrospective nature of the study and all the procedures being performed were part of the routine care.

\section{References}

1. Allen VM, Baskett TF, O'Connell CM, McKeen D, Allen AC. Maternal and perinatal outcomes with increasing duration of the second stage of labor. Obstet Gynecol. 2009 Jun;113(6):1248-1258.

2. American College of Obstetricians and Gynecologists (College), Society for Maternal-Fetal Medicine, Caughey AB, Cahill AG, Guise J-M, Rouse DJ. Safe prevention of the primary cesarean delivery. Am J Obstet Gynecol. 2014 Mar;210(3):179-193.

3. Zipori Y, Grunwald O, Ginsberg Y, Beloosesky R, Weiner Z. The impact of extending the second stage of labor to prevent primary cesarean delivery on maternal and neonatal outcomes. Am J Obstet Gynecol. 2019;220(2):191.e1-191.e7. 
4. Grantz KL, Sundaram R, Ma L, Hinkle S, Berghella V, Hoffman MK, et al. Reassessing the duration of the second stage of labor in relation to maternal and neonatal morbidity. Obstet Gynecol. 2018;131(2):345-353.

5. Alexander JM, Leveno KJ, Rouse DJ, Landon MB, Gilbert S, Spong CY, et al. Comparison of maternal and infant outcomes from primary cesarean delivery during the second compared with first stage of labor. Obstet Gynecol. 2007 Apr;109(4):917-921.

6. Tuuli MG, Liu L, Longman RE, Odibo AO, Macones GA, Cahill AG. Infectious morbidity is higher after second-stage compared with first-stage cesareans. Am J Obstet Gynecol. 2014 Oct;211(4):410.e16.

7. Sung JF, Daniels KI, Brodzinsky L, El-Sayed YY, Caughey AB, Lyell DJ. Cesarean delivery outcomes after a prolonged second stage of labor. Am J Obstet Gynecol. 2007 Sep;197(3):306.e1-5.

8. Dupuis $\mathrm{O}$, Ruimark $\mathrm{S}$, Corinne $\mathrm{D}$, Simone T, André $\mathrm{D}$, René-Charles R. Fetal head position during the second stage of labor: comparison of digital vaginal examination and transabdominal ultrasonographic examination. Eur J Obstet Gynecol Reprod Biol. 2005 Dec 1;123(2):193-197.

9. Buchmann EJ, Libhaber E. Accuracy of cervical assessment in the active phase of labour. BJOG. 2007 Jul;114(7):833-837.

10. Barbera AF, Pombar X, Perugino G, Lezotte DC, Hobbins JC. A new method to assess fetal head descent in labor with transperineal ultrasound. Ultrasound Obstet Gynecol. 2009 Mar;33(3):313-319.

11. Torkildsen EA, Salvesen KÅ, Eggebø TM. Prediction of delivery mode with transperineal ultrasound in women with prolonged first stage of labor. Ultrasound Obstet Gynecol. 2011 Jun;37(6):702-708.

12. Tutschek B, Torkildsen EA, Eggeb $\varnothing$ TM. Comparison between ultrasound parameters and clinical examination to assess fetal head station in labor. Ultrasound Obstet Gynecol. 2013 Apr;41(4):425-429.

13. Eggebø TM, Wilhelm-Benartzi C, Hassan WA, Usman S, Salvesen KA, Lees CC. A model to predict vaginal delivery in nulliparous women based on maternal characteristics and intrapartum ultrasound. Am J Obstet Gynecol. 2015 Sep;213(3):362.e1-6.

14. Kasbaoui S, Séverac F, Aïssi G, Gaudineau A, Lecointre L, Akladios C, et al. Predicting the difficulty of operative vaginal delivery by ultrasound measurement of fetal head station. Am J Obstet Gynecol. 2017 Jan 30;216(5):507.e1-507.e9.

15. Kahrs BH, Usman S, Ghi T, Youssef A, Torkildsen EA, Lindtjørn E, et al. Sonographic prediction of outcome of vacuum deliveries: a multicenter, prospective cohort study. Am J Obstet Gynecol. 2017 Mar 19;217(1):69.e1-69.e10. 
16. Chor CM, Poon LCY, Leung TY. Prediction of labor outcome using serial transperineal ultrasound in the first stage of labor. J Matern Fetal Neonatal Med. 2019 Jan;32(1):31-37.

17. Chaemsaithong P, Kwan AHW, Tse WT, Lim WT, Chan WWY, Chong KC, et al. Factors that affect ultrasound-determined labor progress in women undergoing induction of labor. Am J Obstet Gynecol. 2019 Feb 5;220(6):592.e1-592.e15.

18. Gillor M, Levy R, Barak O, Ben Arie A, Vaisbuch E. Can assessing the angle of progression before labor onset assist to predict vaginal birth after cesarean?: A prospective cohort observational study. J Matern Fetal Neonatal Med. 2020 Jun 10;1-8.

19. Chan WWY, Chaemsaithong P, Lim WT, Tse AWT, Kwan AHW, Leung TY, et al. Pre-Induction Transperineal Ultrasound Assessment for the Prediction of Labor Outcome. Fetal Diagn Ther. 2019;45(4):256-267.

20. Sainz JA, García-Mejido JA, Aquise A, Borrero C, Bonomi MJ, Fernández-Palacín A. A simple model to predict the complicated operative vaginal deliveries using vacuum or forceps. Am J Obstet Gynecol. 2019;220(2):193.e1-193.e12.

21. Hassan WA, Eggebø T, Ferguson M, Gillett A, Studd J, Pasupathy D, et al. The sonopartogram: a novel method for recording progress of labor by ultrasound. Ultrasound Obstet Gynecol. 2014 Feb;43(2):189-194.

22. Yuce T, Kalafat E, Koc A. Transperineal ultrasonography for labor management: accuracy and reliability. Acta Obstet Gynecol Scand. 2015 Jul;94(7):760-765.

23. Barak O, Levy R, Flidel O, Zaks S, Gillor M, Hagay Z, et al. The Routine Use of Intrapartum Ultrasound in Clinical Decision-Making during the Second Stage of Labor - Does It Have Any Impact on Delivery Outcomes? Gynecol Obstet Invest. 2018;83(1):9-14.

24. Sherer DM, Miodovnik M, Bradley KS, Langer O. Intrapartum fetal head position I: comparison between transvaginal digital examination and transabdominal ultrasound assessment during the active stage of labor. Ultrasound Obstet Gynecol. 2002 Mar;19(3):258-263.

25. Sherer DM, Miodovnik M, Bradley KS, Langer O. Intrapartum fetal head position II: comparison between transvaginal digital examination and transabdominal ultrasound assessment during the second stage of labor. Ultrasound Obstet Gynecol. 2002 Mar;19(3):264-268.

26. Akmal S, Tsoi E, Kametas N, Howard R, Nicolaides KH. Intrapartum sonography to determine fetal head position. J Matern Fetal Neonatal Med. 2002 Sep;12(3):172-177.

27. Bellussi F, Ghi T, Youssef A, Salsi G, Giorgetta F, Parma D, et al. The use of intrapartum ultrasound to diagnose malpositions and cephalic malpresentations. Am J Obstet Gynecol. 2017 Jul 22;217(6):633641. 
28. Gustapane S, Malvasi A, Tinelli A. The use of intrapartum ultrasound to diagnose malpositions and cephalic malpresentations. Am J Obstet Gynecol. 2018 Feb 1;218(5):540-541.

29. Ghi T, Farina A, Pedrazzi A, Rizzo N, Pelusi G, Pilu G. Diagnosis of station and rotation of the fetal head in the second stage of labor with intrapartum translabial ultrasound. Ultrasound Obstet Gynecol. 2009 Mar;33(3):331-336.

30. Chan YTV, Ng VKS, Yung WK, Lo TK, Leung WC, Lau WL. Relationship between intrapartum transperineal ultrasound measurement of angle of progression and head-perineum distance with correlation to conventional clinical parameters of labor progress and time to delivery. J Matern Fetal Neonatal Med. 2015 Aug;28(12):1476-1481.

31. Hamilton EF, Simoneau G, Ciampi A, Warrick P, Collins K, Smith S, et al. Descent of the fetal head (station) during the first stage of labor. Am J Obstet Gynecol. 2016 Mar;214(3):360.e1-6.

32. Dückelmann AM, Bamberg C, Michaelis SAM, Lange J, Nonnenmacher A, Dudenhausen JW, et al. Measurement of fetal head descent using the "angle of progression" on transperineal ultrasound imaging is reliable regardless of fetal head station or ultrasound expertise. Ultrasound Obstet Gynecol. 2010 Feb;35(2):216-222.

33. Molina FS, Terra R, Carrillo MP, Puertas A, Nicolaides $\mathrm{KH}$. What is the most reliable ultrasound parameter for assessment of fetal head descent? Ultrasound Obstet Gynecol. 2010 Oct;36(4):493-499.

34. Ghi T, Youssef A, Maroni E, Arcangeli T, De Musso F, Bellussi F, et al. Intrapartum transperineal ultrasound assessment of fetal head progression in active second stage of labor and mode of delivery. Ultrasound Obstet Gynecol. 2013 Apr;41(4):430-435.

35. Głuszak M, Fracki S, Wielgoś M, Wegrzyn P. [Methods of evaluating labor progress in contemporary obstetrics]. Ginekol Pol. 2013 Aug;84(8):709-713.

36. Ghi T, Maroni E, Youssef A, Morselli-Labate AM, Paccapelo A, Montaguti E, et al. Sonographic pattern of fetal head descent: relationship with duration of active second stage of labor and occiput position at delivery. Ultrasound Obstet Gynecol. 2014 Jul;44(1):82-89.

37. Głuszak M, Dziadecki W, Wielgoś M, Węgrzyn P. Evaluation of sonographic assessment of the progress of labor. Ginekol Pol. 2015 Feb;86(2):126-131.

38. Nishimura K, Yoshimura K, Kubo T, Hachisuga T. Objective diagnosis of arrested labor on transperineal ultrasound. J Obstet Gynaecol Res. 2016 Jul;42(7):803-809.

39. Wiafe YA, Whitehead B, Venables H, Odoi AT. Sonographic parameters for diagnosing fetal head engagement during labour. Ultrasound. 2018 Feb 7;26(1):16-21. 
40. Tutschek B, Braun T, Chantraine F, Henrich W. A study of progress of labour using intrapartum translabial ultrasound, assessing head station, direction, and angle of descent. BJOG. 2011 Jan;118(1):62-69.

41. Yonetani N, Yamamoto R, Murata M, Nakajima E, Taguchi T, Ishii K, et al. Prediction of time to delivery by transperineal ultrasound in second stage of labor. Ultrasound Obstet Gynecol. 2017 Feb;49(2):246-251.

42. Tutschek B, Braun T, Chantraine F, Henrich W. Re: Prediction of delivery time in second stage of labor using transperineal ultrasound. Ultrasound Obstet Gynecol. 2017;49(5):663-664.

43. Ghi T, Eggeb $\varnothing \mathrm{T}$, Lees $\mathrm{C}$, Kalache K, Rozenberg P, Youssef A, et al. ISUOG Practice Guidelines: intrapartum ultrasound. Ultrasound Obstet Gynecol. 2018;52(1):128-139.

44. Kalache KD, Dückelmann AM, Michaelis SAM, Lange J, Cichon G, Dudenhausen JW. Transperineal ultrasound imaging in prolonged second stage of labor with occipitoanterior presenting fetuses: how well does the "angle of progression" predict the mode of delivery? Ultrasound Obstet Gynecol. 2009 Mar;33(3):326-330.

45. Gilboa Y, Kivilevitch Z, Spira M, Kedem A, Katorza E, Moran O, et al. Head progression distance in prolonged second stage of labor: relationship with mode of delivery and fetal head station. Ultrasound Obstet Gynecol. 2013 Apr;41(4):436-441.

46. Dall'Asta A, Angeli L, Masturzo B, Volpe N, Schera GBL, Di Pasquo E, et al. Prediction of spontaneous vaginal delivery in nulliparous women with a prolonged second stage of labor: the value of intrapartum ultrasound. Am J Obstet Gynecol. 2019 Oct 4;221(6):642.e1-642.e13.

47. Masturzo B, De Ruvo D, Gaglioti P, Todros T. Ultrasound imaging in prolonged second stage of labor: does it reduce the operative delivery rate? J Matern Fetal Neonatal Med. 2014 Oct;27(15):15601563.

48. Hjartardottir H, Lund SH, Benediktsdottir S, Geirsson RT, Eggeb $\varnothing$ TM. Fetal descent in nulliparous women assessed by ultrasound: a longitudinal study. Am J Obstet Gynecol. 2020 Oct 8;

\section{Tables}


Table 1

Maternal and neonatal demographic and clinical characteristics by the mode of delivery.

Delivery mode

p-value

$$
\begin{array}{ll}
\text { Vaginal delivery }(n=158) & \text { Cesarean delivery } \\
(n=33)
\end{array}
$$

Demographic characteristics

\begin{tabular}{|llll|}
\hline Age (years) & $29.1 \pm 4.3$ & $29.3 \pm 5.2$ & 0.99 \\
\hline BMI $\left(\mathrm{kg} / \mathrm{m}^{2}\right)$ & $23.28 \pm 4.52$ & $25.52 \pm 6.02$ & 0.029 \\
\hline Gestational age (weeks) & $40.02 \pm 1.11$ & $40.16 \pm 1.39$ & 0.325 \\
\hline Birth weight (grams) & $3355 \pm 380$ & $3491 \pm 481$ & 0.14 \\
\hline Clinical data & & $51.5(17)$ & 0.036 \\
\hline Labor induction & $32.2(51)$ & $87.9(29)$ & 0.032 \\
\hline Oxytocin augmentation & $69.6(110)$ & $45.5(15)$ & $<0.001$ \\
\hline Occiput posterior* & $9.5(15)$ & $250 \pm 33$ & 0.093 \\
\hline Second stage duration (minutes) & $239 \pm 29$ & & 0.35 \\
\hline Clinical outcomes & & $0(0)$ & 0.14 \\
\hline Perineal tear 3rd or 4th degree & $2.5(4($ & $24.2(8)$ & 0.90 \\
\hline Intrapartum fever $\geq 38^{\circ} \mathrm{C}$ & $14.5(23)$ & $12.1(4)$ & 0.89 \\
\hline PPH & $11.3(18)$ & $0(0)$ & 0.89 \\
\hline Apgar score (5 minute) $\leq 7$ & $2.5(4)$ & $0(0)$ & \\
\hline neonatal intensive care unit admissions & $2.5(4($ & & \\
\hline Data presented as mean \pm SD or percentage $(\mathrm{n})$. & & \\
\hline$*$ Occiput posterior diagnosed by ultrasound at prolonged second stage. & \\
\hline BMI body mass index, $P P H$ postpartum hemorrhage & & \\
\hline
\end{tabular}


Table 2

Logistic regression analysis of clinical and ultrasound characteristics among 161 women with prolonged second stage of labor with nonocciput posterior (OP) head position (as assessed by ultrasound)

\begin{tabular}{|llll|}
\hline & OR & $95 \%$ Cl & p-value \\
\hline AOP measurement & 1.070 & $1.031-1.111$ & $<0.001$ \\
\hline Birth weight (grams) & 0.998 & $0.997-1.000$ & 0.041 \\
\hline Second stage duration (hours) & 1.000 & $1.000-1.001$ & 0.082 \\
\hline Oxytocin augmentation & 0.353 & $0.063-1.982$ & 0.24 \\
\hline Labor induction & 0.231 & $0.064-0.836$ & 0.026 \\
\hline Gestational age (weeks) & 0.593 & $0.334-1.053$ & 0.075 \\
\hline BMl & 1.028 & $0.881-1.199$ & 0.73 \\
\hline Age (year) & 0.944 & $0.824-1.082$ & 0.41 \\
\hline AOP angle of progression, BMI body mass index & \\
\hline
\end{tabular}

\section{Figures}

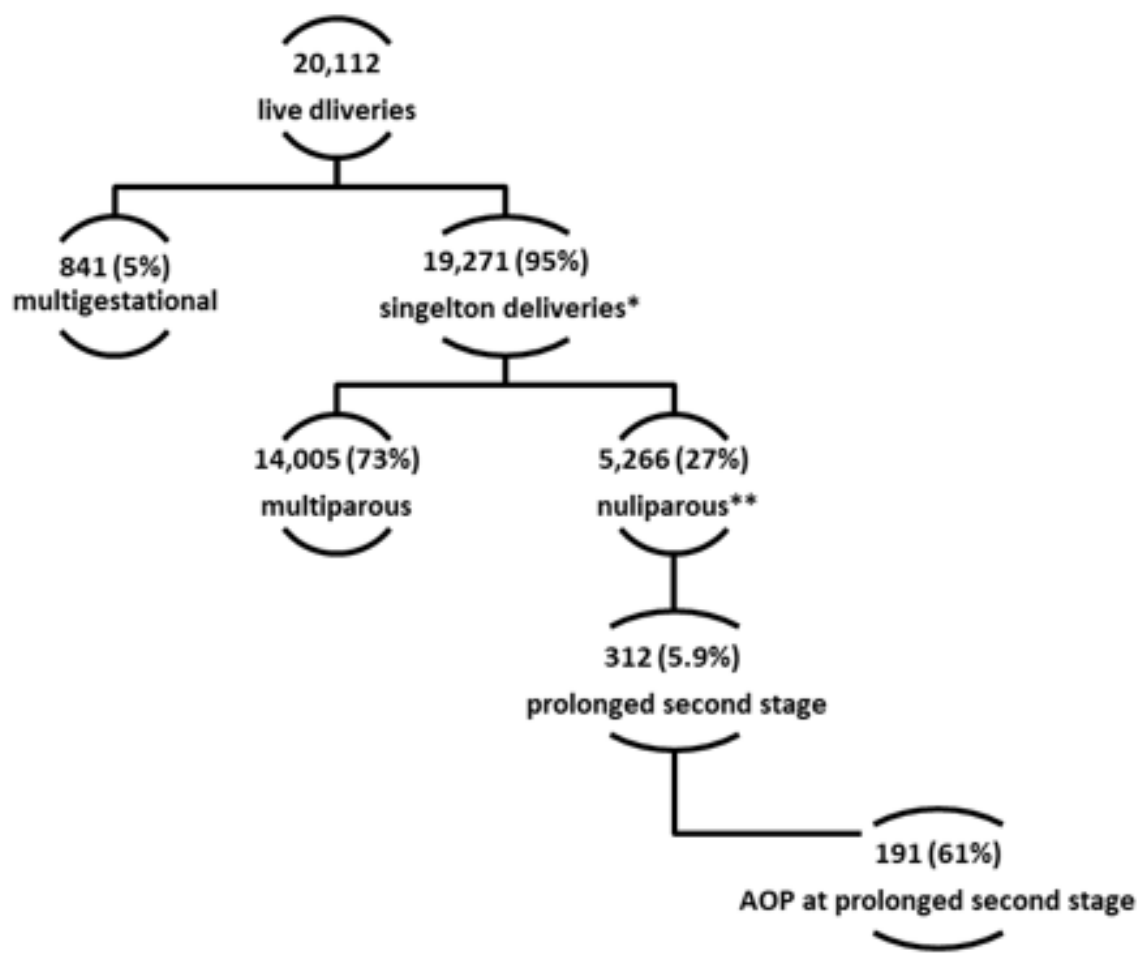

Figure 1

Deliveries during the study period; *SVD: $n=15,249$ (79.2\%), VE: $n=1,045$ (5.4\%), CD: $n=2977$ (15.4\%) **SVD: $n=3,730$ (70.9\%), VE: $n=630$ (11.9\%), CD: n=906 (17.2\% ) SVD spontaneous vaginal delivery, VE 
vacuum extraction, $\mathrm{CD}$ cesarean delivery

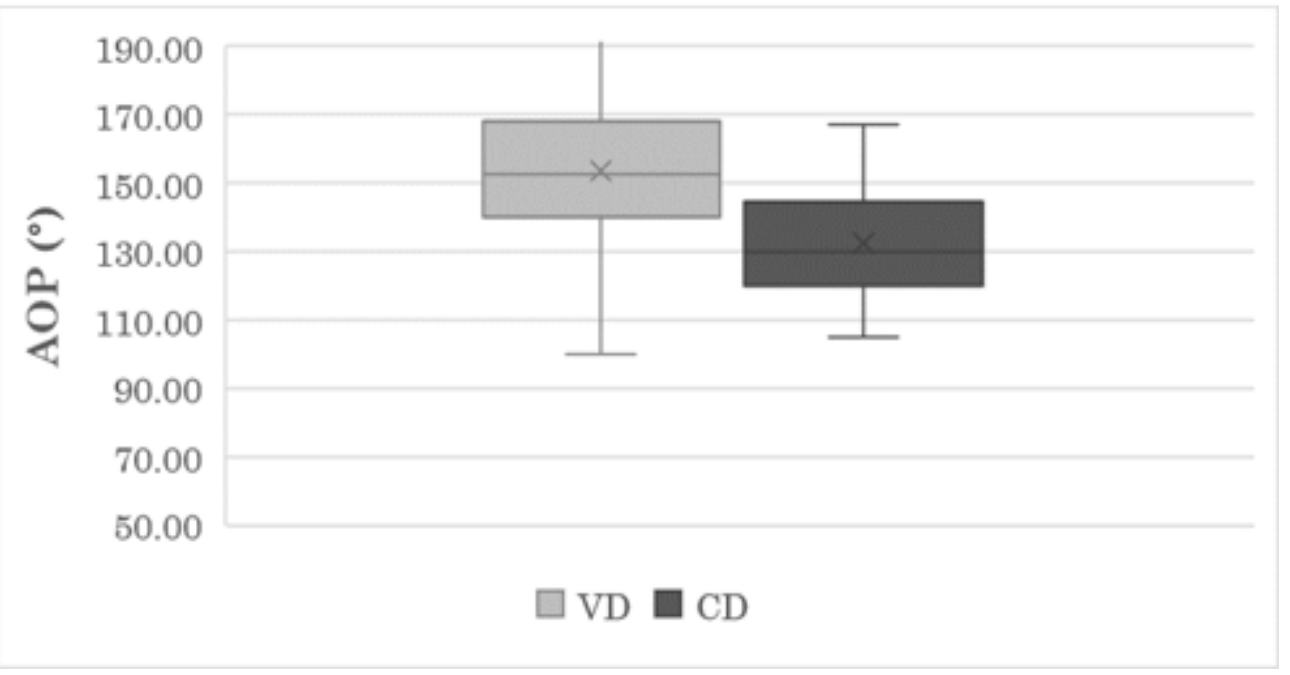

\section{Figure 2}

Mean angle of progression (AOP) documented at prolonged second stage of labor by delivery mode; VD $(n=158)$ : mean $A O P=133^{\circ} \pm 17$ vs. $C D(n=33)$ : mean $A O P=153^{\circ} \pm 19, p<0.001$ VD vaginal delivery, $C D$ cesarean delivery

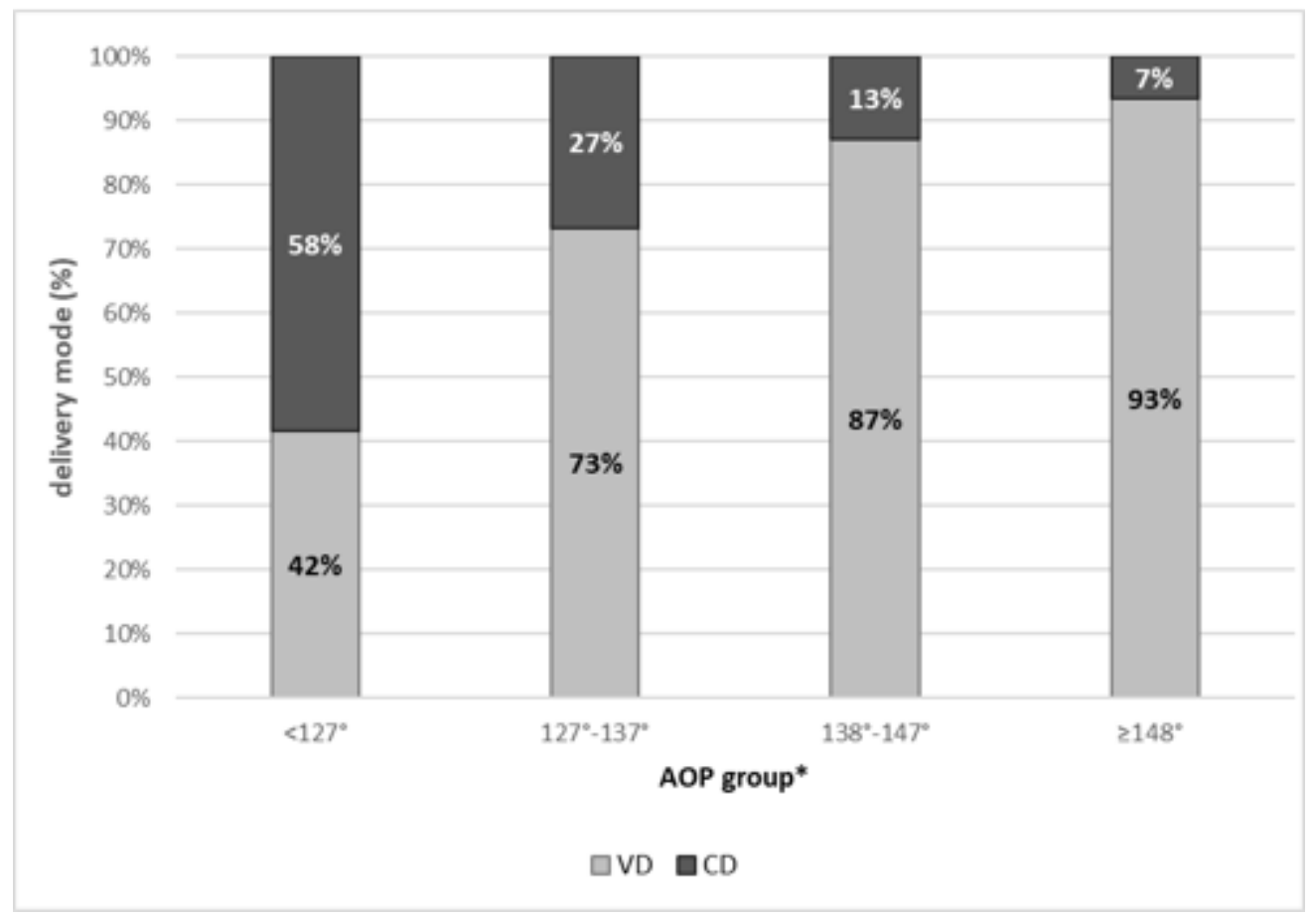

\section{Figure 3}

Mode of delivery by cutoff angle of progression (AOP) documented at prolonged second stage of labor. AOP $<127^{\circ}(n=24)$ VD rate $=42 \%$, AOP $127^{\circ}-137^{\circ}(n=30)$ VD rate $=73 \%$, AOP $138^{\circ}-148^{\circ}(n=31)$ VD rate $=87 \%, A O P \geq 148^{\circ}(n=106), V D$ rate $=93 \% X$ axis refer to $\left.A O P{ }^{\circ}\right)$ CD cesarean delivery, VD vaginal delivery 


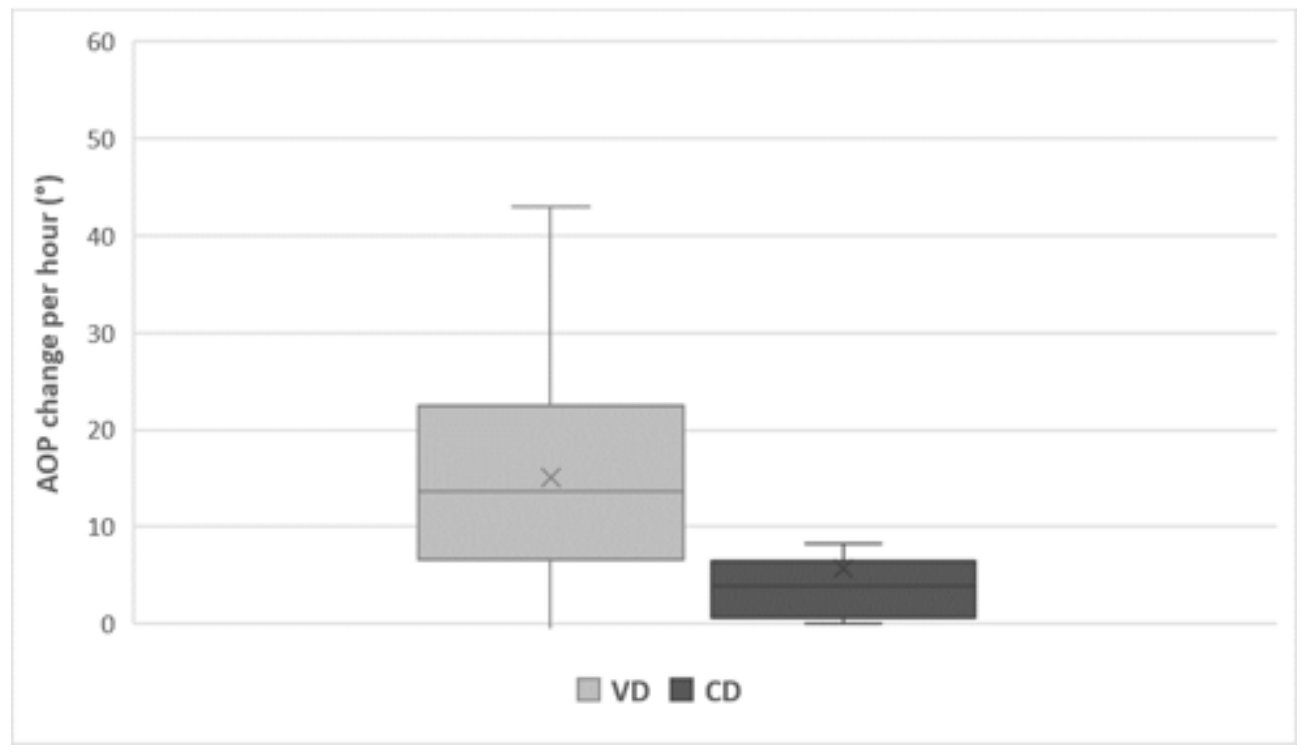

\section{Figure 4}

Mean AOP change per hour from complete dilatation by delivery mode. VD $(n=66)$ mean AOP change per hour $=15.1^{\circ} \pm 11.4$ vs. $C D(n=21)$ mean AOP change per hour $=5.7^{\circ} \pm 6.1^{\circ}$ AOP angle of progression, VD vaginal delivery, $C D$ cesarean delivery

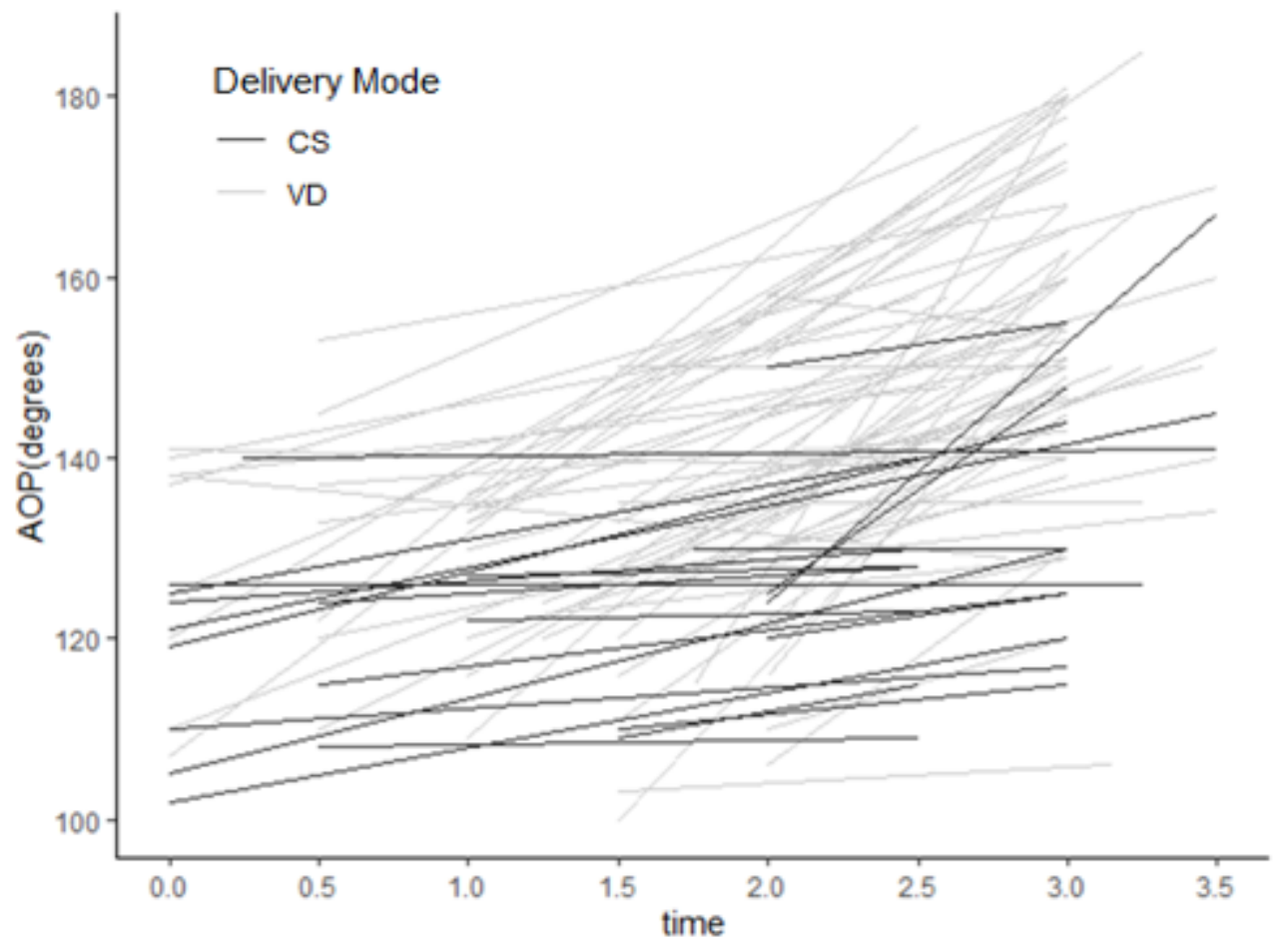

\section{Figure 5}

Individual patterns of fetal head descent measured by AOP in vaginal deliveries $(n=66)$ and cesarean deliveries $(n=21)$. AOP angle of progression, VD vaginal delivery, $C D$ cesarean delivery 


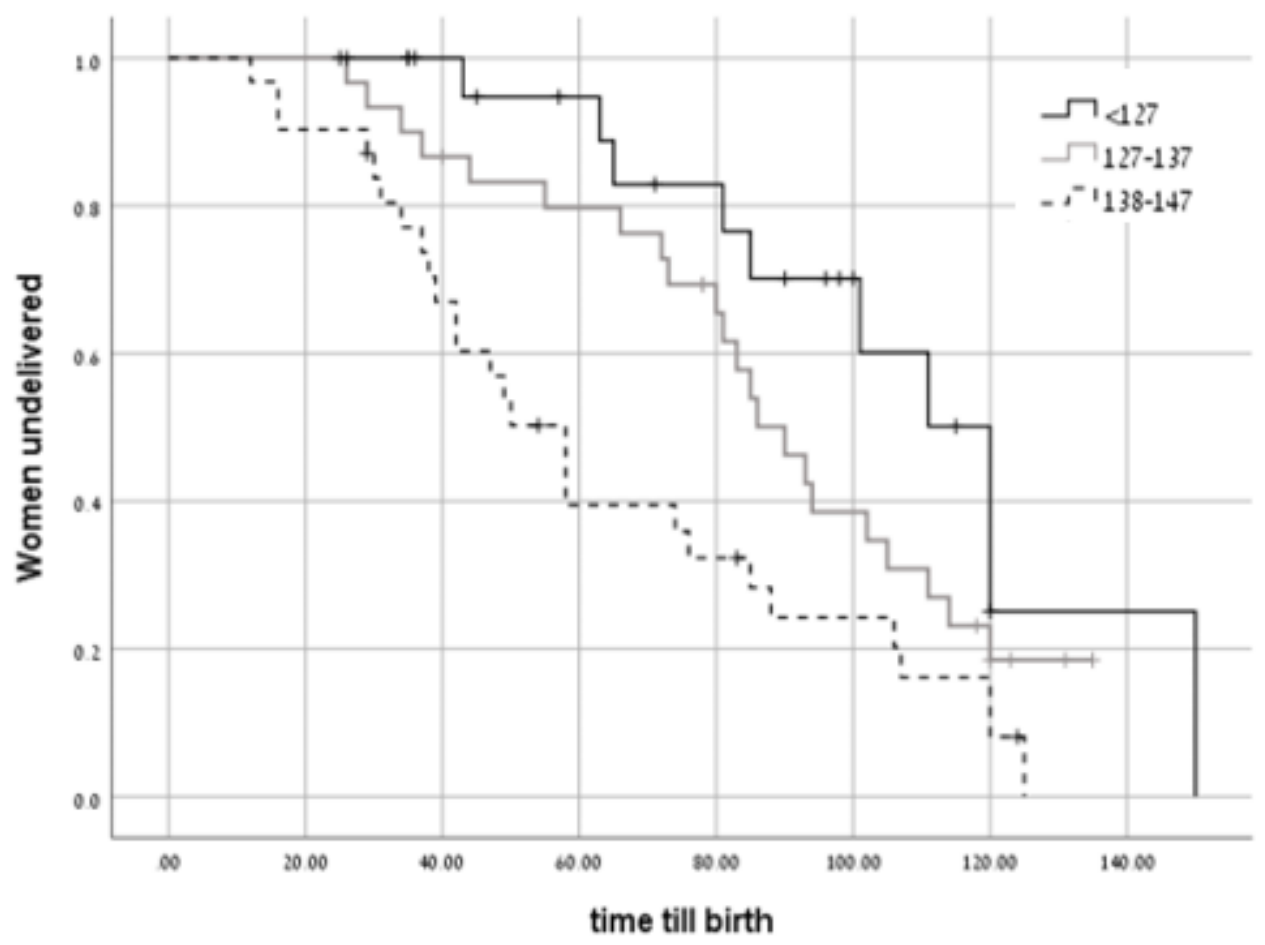

Figure 6

Kaplan-Meier plot of interval time to delivery (minutes) by angle of progression (AOP) documented at prolonged second stage by AOP groups according to the discriminating cutoff suggested by Tutschek et al. $\left(127^{\circ}\right.$ correspond to Station $1 \mathrm{~cm} ; 138^{\circ}$ corresponds to Station $2 \mathrm{~cm}, 148^{\circ}$ corresponds to Station 3 $\mathrm{cm}$ ) [40]. Cases were censored at the time of cesarean delivery(vertical lines).

\section{Supplementary Files}

This is a list of supplementary files associated with this preprint. Click to download.

- Declarations.docx 